\title{
COMPORTAMENTO DE CRIANÇAS E ADOLESCENTES PORTADORES DE DIABETES MELLITUS TIPO 1 NO AMBIENTE ESCOLAR
}

\section{Behavior of children and adolescents with type 1 diabetes mellitus in the school environment}

Caroline Arns-Neumann ${ }^{1, *}$, Camilla Harumi Tabushi', Andreia Araújo Porchat Leão ${ }^{1}$, Camilla Kapp Fritz ${ }^{1}$, Andrea Cristina de Souza Silva ${ }^{1}$, Gabriela Carvalho Kraemer ${ }^{1}$, Adriane de Andre Cardoso-Demartini ${ }^{1}$, Lylian Christine Stansky ${ }^{1}$, Monica Nunes Lima Cat ${ }^{1}$, Suzana Nesi-França

${ }^{1}$ Universidade Federal do Paraná, Departamento de Pediatria - Curitiba - Paraná - Brasil.

\section{RESUMO}

Introdução: Diabetes mellitus tipo 1 (DM1) é uma doença cujo controle é desafiador, incluindo os cuidados na escola. Objetivo: Avaliar o comportamento e identificar problemas no ambiente escolar. Métodos: Estudo transversal com aplicação de questionário aos pacientes e cuidadores atendidos em serviço de endocrinopediatria. Resultados: Foram avaliados 129 pacientes, 114 do ensino fundamental/médio (EFM) e 15 da educação infantil (EI), com medianas de idade de 11 anos (2-17 anos) e de tempo de doença de 4 anos (0,2-14 anos). Entre os alunos do EFM, 40\% aplicavam insulina na escola, entre eles $20 \%$ aplicavam no banheiro, $69,6 \%$ usavam caneta e apenas um, seringa $(p<0,001)$. A hemoglobina glicada foi maior entre os que já haviam reprovado na escola $(p<0,01)$. A maior parte $(93,9 \%)$ lanchava na escola, apenas $37,7 \%$ recebiam lanche para DM1, mais frequente na escola municipal ( $p<0,001)$. Em relação à hipoglicemia, 78,1\% já apresentaram pelo menos um episódio na escola, 63,2\% relataram que os professores não conheciam o tratamento, 39,5\% não levavam glicosímetro para escola, 16,7\% mediam a glicemia antes da educação física e 51,7\% sentiam medo de passar mal na escola. Dos alunos da El, 66,7\% recebiam insulina na escola. Apenas 2 pacientes tinham glucagon na escola para hipoglicemia grave. Conclusão: Observou-se despreparo das instituições para aplicação de insulina, fornecimento de cardápio individualizado, e para urgências que demandam assistência imediata como hipoglicemia. As dificuldades de controle do DM1 na escola justificam a necessidade de desenvolver estratégias que promovam melhor qualidade de vida no ambiente escolar aos portadores de DM1.

Palavras-chave: Diabetes Mellitus Tipo 1. Comportamento. Serviços de Saúde Escolar.

\section{ABSTRACT}

Introduction: Type 1 diabetes mellitus (T1DM) is disease whose control represents a challenge, including school care. Objective: To evaluate behavior and identify problems in the school. Methods: Cross-sectional study with questionnaire application to patients and parents attended in a pediatric endocrine center. Results: 129 patients were evaluated, 114 from elementar/middle/high school (EMHS), 15 from pre-school (PS), with a median age of 11 years (2-17 years) and 4 years (0,2-14 years) of disease. Among EMHS students, $40 \%$ receive ply insulin at school, $(20 \%$ in the toilet), $69.6 \%$ use a pen and only one, syringe ( $p<0.001)$. Glycated hemoglobin was higher among those who had already school failure $(p<0.01)$. The majority $(93.9 \%)$ had snack time at school, only $37.7 \%$ receive a specific snack for T1DM, with higher frequency in the municipal school $(\mathrm{p}<0.001)$. Regarding hypoglycemia, $78.1 \%$ have had at least one episode at school, $63.2 \%$ reported that teachers do not know the treatment, $39.5 \%$ do not take the glucometer to school and only $16.7 \%$ measure blood glucose before physical education, $51.7 \%$ feel afraid of being sick at school. Between PS students, $66.7 \%$ receive insulin at school. Only two patients reported availability of glucagon at school for severe hypoglycemia. Conclusion: There is evident unpreparedness of the institutions, to ensure an adequate place for insulin administration, to provide individualized menu, and for emergencies that require immediate assistance such as hypoglycemia. The difficulties of controlling T1DM in school justify the need to develop strategies that promote better quality of life at school for patients with T1DM.

Keywords: Diabetes Mellitus, Type 1, Behavior. Schools

\footnotetext{
* Autor correspondente : Caroline Arns-Neumann

E-mail: carolinearnsneumann@gmail.com
} 


\section{INTRODUÇÃO}

O diabetes mellitus tipo 1 (DM1) é uma das doenças crônicas mais comuns da infância e adolescência, caracterizada pela produção deficiente de insulina, resultando em hiperglicemia e necessitando de administração diária de insulina. ${ }^{1}$ A Associação Americana de Diabetes discute a complexidade da condição que requer estratégias multifatoriais de redução de risco. ${ }^{2}$

O tratamento dessa doença crônica demanda envolvimento ativo do paciente e cuidadores, com o objetivo de manter o melhor controle glicêmico possível, evitar complicações em curto, médio e longo prazo e permitir o desenvolvimento psicológico e emocional adequado. ${ }^{3}$ Estudos mostraram claramente que o controle glicêmico rigoroso a partir do diagnóstico da doença pode postergar ou mesmo evitar complicações vasculares crônicas relacionadas ao $\mathrm{DM} 1 .^{4}$

O tratamento envolve reconhecimento dos sintomas, mudanças de estilo de vida, gerenciamento de tarefas relacionadas à administração de insulina, automonitorização da glicose sanguínea e orientação nutricional e sobre prática de exercícios. ${ }^{5}$ Nesse sentido, o cuidado diário do DM1 apresenta inúmeros desafios para alcançar controle metabólico satisfatório, o que inclui o tempo que a criança está na escola, em que precisam de apoio individualizado adequado para aidade.

Segundo Leite et al. (2008), o paciente diabético tipo 1 e seu núcleo familiar e social, somado à participação da escola, devem ser treinados para administrar o autocuidado em sua rotina diária da melhor maneira possível. O estudo conclui, ainda, que a melhora no autocuidado diminui o valor de hemoglobina glicada, reduzindo o risco de complicações.

A escola é parte essencial na vida de crianças e adolescentes e promover o envolvimento e conhecimento sobre o DM1 dos professores e funcionários das instituições de ensino pode auxiliar não só no controle da doença e suas complicações, como também na aceitação da doença.

$\mathrm{O}$ atual estudo teve como objetivo conhecer o comportamento e mensurar as dificuldades de crianças e adolescentes portadores de DM1 no ambiente escolar, que são atendidos num serviço de referência em endocrinologia pediátrica.

\section{MATERIAIS E MÉTODOS}

Estudo transversal com aplicação de questionários aos portadores de DM1 e aos seus cuidadores atendidos em serviço de referência em endocrinologia pediátrica. A população atendida nesse serviço corresponde a cerca de 350 crianças e adolescentes de Curitiba, região metropolitana e interior do estado do Paraná. Os pacientes comparecem periodicamente para consultas de rotina (a cada 3 meses) e foram avaliados nos dias de consulta do ambulatório de DM1. O estudo foi aprovado pelo Comitê de Ética em Pesquisa em Seres Humanos.

Foram aplicados dois tipos de questionários: um para crianças de 0 a 6 anos (que analisou a resposta dos cuidadores), e outro para crianças a partir dos 6 anos. A amostra foi selecionada por conveniência, com aplicação dos questionários a todos os pacientes que compareceram à consulta médica no período da pesquisa, frequentavam escola e concordaram em participar da pesquisa. Os pacientes responderam o questionário enquanto aguardavam a consulta médica, após explicação pelos pesquisadores dos objetivos, riscos e benefícios da pesquisa e após assinatura do Termo de Consentimento Livre e Esclarecido (TCLE) pelos pais e do Termo de Assentimento Livre e Esclarecido (TALE) pelos pacientes com mais de 12 anos. Foram abordadas questões como histórico da doença e escolar do paciente (14 perguntas), terapia insulínica na escola (9 perguntas), conhecimento da instituição quanto à doença (12 perguntas), alimentação no período escolar (4 perguntas) e cuidados durante a atividade física na escola.

Além disso, foi realizada coleta de dados dos prontuários dos pacientes referente ao controle da doença por meio da média anual de hemoglobina glicada fração A1c (HbA1c). Os dados coletados foram digitados em planilha eletrônica (Microsoft Excel $^{\circ}$ 2010), conferidos e exportados para o programa Statistica 10.0 (Statsoft ${ }^{\circledR}$ ). Na análise estatística foram aplicados os testes qui-quadrado de Pearson/Yates, teste $t$ de Student e de Mann-Whitney. Para todos os testes foi considerado o nível de significância de $5 \%{ }^{6}$

\section{RESULTADOS}

Foram avaliados 129 questionários, sendo 15 de alunos da Educação Infantil e 114 de alunos do Ensino Fundamental ou Médio. A mediana de idade foi de 11 anos (2-17 anos), mediana de idade ao diagnóstico de 5 anos (0,5-13 anos) e mediana de tempo de doença de 4 anos (0,2-14 anos). As características dos participantes do estudo estão apresentadas na tabela 1 .

\section{Pacientes da Educação Infantil}

Entre os 15 pacientes da educação infantil, 9 eram do sexo feminino e 6 do sexo masculino, com mediana de idade de 5 anos (2-5 anos), mediana de idade ao diagnóstico de 2 anos (1-5 anos) e mediana de tempo de doença de 2 anos (0,3-4 anos). A maior parte (80\%) estudava em escola municipal.

Todos os pacientes foram internados no momento do diagnóstico e cinco deles tiveram pelo menos um novo internamento. A maioria (66,7\%) usava insulina glargina e ultrarrápida, $73 \%$ (11) usavam caneta, $20 \%$ (3) seringa e 
Tabela 1. Características dos participantes do estudo.

\begin{tabular}{lcc}
\hline Características & $\mathbf{n}$ & $\%$ \\
\hline Sexo & & \\
Feminino & 66 & 51,2 \\
Masculino & 63 & 48,8 \\
Escolaridade & & \\
Educação Infantil & 15 & 11,6 \\
Ensino Fundamental e Médio & 114 & 88,4 \\
Tipo de escola & & \\
Estadual & 52 & 40,3 \\
Municipal & 62 & 48,1 \\
Privada & 15 & 11,6 \\
Internado ao diagnóstico & & \\
Sim & 125 & 96,9 \\
Não & 4 & 3,1 \\
Controle da doença & & \\
HbA1c<7,5\% & 16 & 12,4 \\
HbA1c>7,5\% & 113 & 87,6 \\
\hline
\end{tabular}

Nota: $\mathrm{HbA1c}=$ Média anual de hemoglobina glicada

$7 \%$ (1) sistema de infusão contínua de insulina. Quanto a aplicação de insulina na escola, mais da metade (66,7\%) dos cuidadores relataram aplicar insulina na escola, sendo realizada pelos professores da instituição (50\%), pais (30\%), coordenador da escola ( $10 \%$ ) ou pedagoda (10\%).

No que se refere a hipoglicemia, $60 \%$ já apresentaram episódios na escola. Quanto ao manejo, 46,7\% dos cuidadores relataram que a escola telefonava para os pais em caso de sintomas de hipoglicemia ou hiperglicemia e $46,7 \%$ mediam a glicemia e tratavam a complicação. Nenhuma escola dispunha de glucagon para casos de hipoglicemia grave. Apesar de todas as escolas estarem cientes do diagnóstico nem todos os professores $(46,7 \%)$ conheciam os sintomas de hipoglicemia.

Quanto ao comportamento na escola, 93\% (14) dos pais relataram não perceber nenhum tipo de exclusão devido à doença. $O$ fornecimento de lanche era feito por $93,3 \%$ das escolas, porém $20 \%$ destas não forneciam alimentação especial para diabéticos.

A média anual de $\mathrm{HbA} 1 \mathrm{c}$ foi de $8,7 \pm 1,3 \%$, sendo que $26,7 \%$ (4) estavam abaixo de $7,5 \%$, o que indicaria controle adequado da doença ${ }^{7}$.

\section{Pacientes do ensino fundamental e médio}

Entre os 114 pacientes do ensino fundamental e médio, 50\% (57) eram do sexo feminino. A mediana de idade deste grupo foi de 11 anos (5-16 anos), com mediana de idade ao diagnóstico de 6 anos (0,5-13 anos) e mediana de tempo de doença de 4 anos (0,2-14 anos). A maior parte dos pacientes $(96,5 \%)$ relatou ter sido internado no diagnóstico de DM1. A mediana de internamentos após o diagnóstico foi de 1 internamento ( 0 a 17 internamentos), sendo que $71(62,3 \%)$ não foram novamente internados após o diagnóstico.

A maioria (89,5\%) frequentava escolas públicas, sendo $51 \%$ escola estadual e $49 \%$ municipal. Na autoavaliação do rendimento escolar, $82(71,9 \%)$ consideraram ter um bom rendimento, $26(22,8 \%)$ regular e $6(5,3 \%)$ ruim. Apesar de a maior parte $(84,2 \%)$ não ter relacionado o rendimento escolar com a doença, 20,2 \% (23) já tinham sofrido reprovação escolar e a média de $\mathrm{HbA1c}$ foi maior entre aqueles que tiveram alguma reprovação na escola $(p<0,01)$.

Referente a administração de insulina, $58,4 \%$ aplicavam insulina glargina e ultrarrápida, $27,4 \%$ insulina $\mathrm{NPH}$ e regular, $13,5 \%$ sistema de infusão contínua de insulina e 2,6\% insulina NPH e ultrarrápida, sendo aplicada por $71,1 \%$ dos pacientes com caneta, $17,5 \%$ seringa e $11,4 \%$ sistema de infusão contínua de insulina. A aplicação de insulina no período escolar foi relatada por $40,3 \%$ dos pacientes, apesar de $93,9 \%$ lancharem na escola. A aplicação era realizada na maior parte das vezes pelos pais $(32,6 \%)$ ou pelo próprio paciente (63\%), geralmente sem supervisão (57,7\%). Entre os locais de aplicação estavam a secretaria (28\%), o banheiro (20\%) e a sala de aula (20\%) (Figura 1). Na presente amostra apenas o fato de não usar seringa e usar sistema de infusão contínua de insulina foram associados a maior probabilidade de aplicar insulina na escola $(p<0,001)$. (Tabela 2$)$.

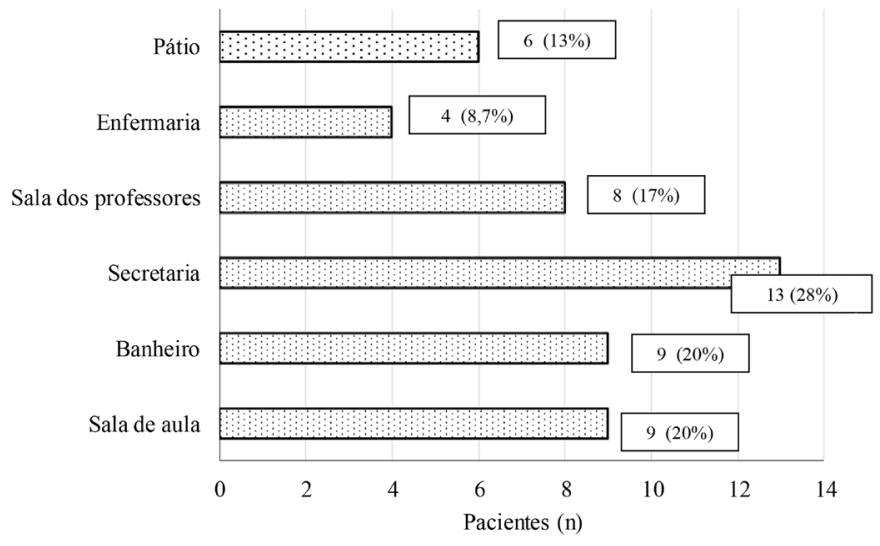

Figura 1. Local de aplicação de insulina na escola.

Apesar de $99,1 \%$ das escolas estarem cientes do diagnóstico, $37,7 \%$ forneciam o lanche especial para diabéticos, sendo que esse fornecimento era feito com maior frequência pela escola municipal $(p<0,001)$ (Figura 2). Apenas $38,6 \%$ realizavam o método de contagem decarboidratos.

No que se refere à hipoglicemia, 78,1\% já haviam apresentado pelo menos um episódio na escola e $66,7 \%$ 
Tabela 2. Fatores associados a aplicação de insulina na escola.

\begin{tabular}{lccc}
\hline Características & Aplica insulina $\mathbf{n}(\%)$ & Não aplica insulina $\mathbf{n}(\%)$ & p \\
\hline Vergonha de contar que tem diabetes & & & \\
Sim & $6(25 \%)$ & $18(75 \%)$ & $0,13^{*}$ \\
Não & $40(44,4 \%)$ & $50(55,6 \%)$ & \\
Vergonha de aplicar insulina na escola & & & \\
Sim & $8(17,4 \%)$ & $8(17,4 \%)$ & $0,06^{*}$ \\
Não & $38(82,6 \%)$ & $44(64,7 \%)$ & $0,93^{*}$ \\
Usa caneta & & & \\
Sim & $32(69,6 \%)$ & $49(72,1 \%)$ & $<0,001^{*}$ \\
Não & $14(30,4 \%)$ & $19(27,9 \%)$ & \\
Usa seringa & & $19(27,9 \%)$ & $<0,001^{*}$ \\
Sim & $1(2,2 \%)$ & $49(72,1 \%)$ & \\
Não & $45(97,8 \%)$ & $0(0,0 \%)$ & \\
Usa bomba & & $68(100 \%)$ & \\
Sim & $13(28,3 \%)$ & $33(71,7 \%)$ & \\
Não & & & \\
\hline
\end{tabular}

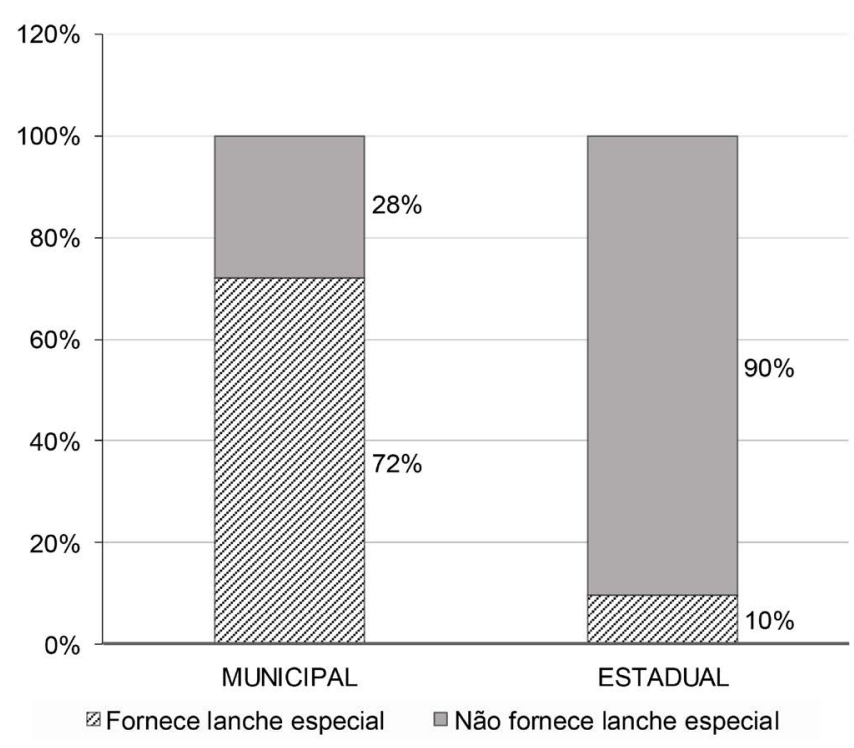

Figura 2. Fornecimento de lanche especial para alunos com DM1

relataram que os professores não sabiam o que fazer nessas situações, 63,2\% tampouco conheciam os sintomas de hipoglicemia. Entre os colegas, $29,8 \%$ conheciam os sintomas de hipoglicemia e $24,6 \%$ sabiam o que fazer em caso de hipoglicemia. Em relação à aferição da glicemia, 39,5\% (45) dos pacientes não levavam o glicosímetro para a escola, o que prejudica o correto diagnóstico dessa situação.

O tratamento para hipoglicemia foi descrito como inapropriado (fruta, biscoito, arroz e feijão, entre outros) em 23,6\% dos casos e 2 dos 114 pacientes relataram ter glucagon disponível na escola em caso de hipoglicemia grave nas crianças.

Os entrevistados responderam que 90,3\% dos professores sabiam do diagnóstico, 91,1\% permitiam sair durante a aula caso não estivessem se sentindo bem, $85,1 \%$ permitiam sair durante a aula para tomar água e $83,3 \%$ permitiam sair para ir ao banheiro.

Referente à educação física, apesar de 94,7\% dos pacientes participarem das aulas de educação física e 81,2\% dos professores de educação física estarem cientes do diagnóstico, apenas $36,6 \%$ sabiam o que fazer em caso de hipoglicemia.

Entre as dificuldades que os pacientes encontravam quanto a doença no âmbito escolar, o medo de passar mal foi o mais prevalente (51,7\%). Além disso, $28,1 \%$ referiu não ter o apoio do colégio, $21 \%$ referiu vergonha de contar que tinham diabetes, $28,1 \%$ referiu vergonha de medir a glicemia ou aplicar a insulina na escola, 8,8\% sentiam-se excluídos por ter diabetes, $8,8 \%$ sofriam bullying, $16,7 \%$ referiu não ter o apoio dos colegas e $20,2 \%$ se sentiam excluídos por receber lanche diferente do oferecido aos colegas.

A média anual de $\mathrm{HbA} 1 \mathrm{c}$ foi de $9,6 \pm 1,7 \%$, sendo que $10,5 \%$ estavam abaixo de $7,5 \%$, o que indicaria controle adequado da doença.

\section{DISCUSSÃO}

O aumento global na incidência de DM1, especialmente em crianças mais jovens, associado às evidências da influência do controle glicêmico na evolução a longo prazo dos pacientes, demandam cada vez mais que as escolas estejam preparadas para proporcionar um ambiente que permita o cuidado adequado relacionado ao diabetes. ${ }^{5}$

Apesar de $99,1 \%$ das escolas dos pacientes investigados neste estudo estarem cientes do diagnóstico, 
o suporte oferecido aos pacientes portadores de DM1 no ambiente escolar é ainda muito frágil e isso fica evidente pelas dificuldades que estes apresentam.

Estudo qualitativo identificou falha na transferência de informações relacionadas à doença dos diretores para os professores. ${ }^{8} \mathrm{O}$ mesmo pode ter ocorrido na presente amostra, em que apesar da maioria das escolas estar ciente do diagnóstico $(99,1 \%)$ nem todos os professores $(90,3 \%)$ sabiam da condição da criança ou sabiam como auxiliar no tratamento das crianças e adolescentes.

As dificuldades relatadas pelos pacientes no presente estudo, como vergonha de medir a glicemia capilar ou aplicar a insulina na escola, discriminação pelo alimento oferecido no lanche ser diferente, falta de informação e compreensão dos professores com limitação de saídas da sala de aula para ir ao banheiro e beber água ou em caso de não estar sentindo-se bem, também foram relatadas em outro estudo. ${ }^{8}$ No estudo DAWN Youth WebTalk Study, uma pesquisa internacional com 6789 participantes de oito países, foi questionado para estudantes com DM1 sobre o apoio fornecido na escola, que foi considerado baixo. A maioria dos indivíduos relatou que os professores precisam estar mais bem informados sobre o diabetes e precisavam ser treinados para lidar com situações emergenciais da doença.'

Andrade et al (2019) identificaram interação significativa entre os fatores psicológicos com o controle glicêmico nos portadores de DM1. ${ }^{10}$ Apesar de na presente amostra o apoio do colégio não ter influenciado significativamente o controle glicêmico, a Sociedade Internacional de Diabetes para Pediatria e Adolescência recomenda exames de rotina para questões psicossociais em crianças e adolescentes com DM1, para identificar a necessidade de aconselhamento especializado. ${ }^{11}$

A maioria dos pacientes não relacionou o rendimento escolar com a doença, o que está em consonância com estudo coorte, que encontrou desempenho escolar semelhante entre diabéticos tipo 1 e seus pares, apesar de apresentarem 3\% a menos de presença na escola. Porém, esse mesmo estudo encontrou associação moderada entre pior controle glicêmico e pior desempenho escolar semelhante ao achado do presente estudo, em que a média de HbA1c foi maior entre aqueles que tiveram alguma reprovação na escola $(p<0,01){ }^{12}$

Cuidado especial deve ser dado à hipoglicemia, uma vez que pode prejudicar o desenvolvimento cerebral e comprometer a qualidade de vida. ${ }^{13} \mathrm{~A}$ maior parte das crianças relatou ter apresentado pelo menos um episódio de hipoglicemia na escola, o que reforça a necessidade de atenção por parte dos professores e colegas frente a essa situação, especialmente os professores de educação física, uma vez que a hipoglicemia é comum durante ou imediatamente após o exercício. Diferentes sociedades orientam a aferição da glicemia pré exercício para prevenir o risco de hipoglicemia, no entanto, no presente estudo apesar da maioria dos professores de educação física saberem do diagnóstico de seus alunos, $63 \%$ dos participantes relatam que os professores não sabiam o que fazer em caso de hipoglicemia e a maioria dos alunos não mediam a glicemia antes da atividade física. ${ }^{14}$

O medo de passar mal, relatado por $51,7 \%$ das crianças, pode ser fator dificultador do controle glicêmico. Estudos apontam que o medo da hipoglicemia esteve associado a maiores valores de $\mathrm{HbA} 1 \mathrm{c}$ e menor qualidade de vida. ${ }^{15}$ A monitorização constante da glicemia é importante para prevenção de episódios de hipoglicemia, ${ }^{16}$ porém é limitada pela vergonha de exposição e medo de danificar o glicosímetro pela falta de local apropriado para aferição. ${ }^{8} \mathrm{Na}$ presente amostra 39,5\% dos pacientes relataram não levar o glicosímetro para a escola.

Nos pacientes da Educação Infantil é comum a escola telefonar para os pais em caso de hipoglicemia. Em apenas $46,7 \%$ dos casos a escola mensurou e corrigiu a hipoglicemia, outro estudo relatou também que em momentos de emergência, a ação da escola limitou-se a chamar os pais. ${ }^{8}$

A alimentação adequada é fundamental para o sucesso no tratamento de portadores de DM1, no entanto, diferentes estudos mostram que os profissionais das escolas não tem conhecimento sobre os cuidados com a alimentação no DM1. Smith et al., desenvolveram um programa educacional sobre diabetes para professores, neste estudo $50 \%$ dos profissionais tinham baixo conhecimentos sobre a alimentação adequada para diabetes, o que demonstra a dificuldade das escolas em identificar as reais necessidades das crianças portadoras de alguma enfermidade. ${ }^{17}$ No presente estudo, a maior parte das escolas da educação infantil (80\%) fornecia lanche especial, porém, nas escolas das crianças acima de 6 anos, apenas $37,7 \%$ forneciam o lanche. $O$ estudo aponta uma lacuna no cuidado com o diabetes, principalmente nas escolas estaduais, com o não cumprimento da lei $n^{\circ} 12.982$, de maio de 2014 que determina o provimento de alimentação escolar adequada aos alunos portadores de estado ou de condição de saúde específica. ${ }^{18}$

Como limitação do estudo, deve-se ressaltar que esta pesquisa abordou somente a visão do paciente e seus responsáveis, não tendo sido avaliada a visão da escola, o que deve ser realizado em estudos futuros para um melhor entendimento do tema.

\section{CONCLUSÕES}

Apesar das escolas terem conhecimento acerca da doença dos alunos, existe um evidente despreparo das instituições, tanto para assegurar um local adequado para aplicações de insulina ou fornecer cardápio individualizado quanto para urgências que demandam assistência imediata como a hipoglicemia, já que a maior parte das escolas não disponibiliza oglucagon. 
O paciente portador de DM1 e seu núcleo familiar e social, somado à participação da escola, devem ser treinados para administrar o autocuidado em sua rotina diária da melhor maneira possível. Além da família, a escola é parte essencial na vida de crianças e adolescentes. Por este motivo, seu envolvimento e conhecimento sobre o DM podem auxiliar não só no controle da doença e complicações, como também na aceitação da doença.

O conhecimento acerca das dificuldades de controle do DM1 no ambiente escolar justifica a necessidade de estudar mais esse tema, para identificar os principais problemas e desenvolver estratégias que minimizem danos e promovam melhor qualidade de vida aos portadores de diabetes no ambiente escolar.

\section{REFERÊNCIAS}

1. Dimeglio LA, Evans-molina C, Oram RA. Seminar Type 1 diabetes. Lancet [Internet]. 2018; 391 (10138):2449-62. Available from: http://dx.doi.org/10.1016/S0140-6736 (18) 31320-5

2. American Diabetes Association (2014) Standards of Medical Care in Diabetes - 2014. Diabetes Care. 37, 1, 14-80.

3. Helgeson VS, Reynolds KA, Siminerio L, Escobar O, Becker D. Parent and adolescent distribution of responsibility for diabetes self-care: links to health outcomes. J Pediatr Psychol. 2008; 33: 497-508

4. Ceriello A, Monnier L, Owens D. Review Glycaemic variability in diabetes : clinical and therapeutic implications. Lancet Diabetes Endocrinol. 2019 Mar;7(3):221-230. Available from: http://dx. doi: 10.1016/S2213-8587(18)30136-0. Epub 2018 Aug 13.

5. Bratina N, Forsander G, Annan F, Wysocki T, Pierce J et al. ISPAD Clinical Practice Consensus Guidelines 2018: Management and support of children and adolescents with type 1 diabetes in school. Pediatr Diabetes . 2018; (July):287-301.

6. R Core Team R: A language and environment for statistical computing. R Foundation for Statistical Computing, Vienna, Austria. 2018 URL https://www.R-project.org/

7. DiMeglio LA, Acerini CL, Codner E, Craig ME, Hofer SE, Pillay K, Maahs DM. ISPAD Clinical Practice Consensus Guidelines 2018: Glycemic control targets and glucose monitoring for children, adolescents, and young adults with diabetes. Pediatr Diabetes. 2018 Oct;19 Suppl 27:105-114.
8. Sparapani VC, Liberatore RDR, Damião EBC, Dantas IRO, Camargo RAA. Children With Type 1 Diabetes Mellitus: Self-Management Experiences in School. Journal of School Health. 2017; 87 (8):623-9.

9. Peyrot M. International DAWN Youth Survey Group. The DAWN Youth WebTalk Study: methods, findings, and implications. Pediatr Diabetes, 2009 Dec; 10 Suppl 13:37-45.

10. Andrade CJDN, Alves CAD. Influence of socioeconomic and psychological factors in glycemic control in young children with type 1 diabetes mellitus. J Pediatr (Rio J). 2019;95(1):48-53.

11. Delamater AM, Wit M De, McDarby V, Malik JA, Hilliard ME, Northam E, et al. ISPAD CLINICAL PRACTICE CONSENSUS GUIDELINES ISPAD Clinical Practice Consensus Guidelines 2018: Psychological care of children and adolescents with type 1 diabetes. 2018; 19 (October):237-49.

12. Cooper MN, McNamara KAR, de Kler NH, Davis EA, Jones TW. School performance in children with type 1 diabetes : a contemporary population-based study. 2014; (6):1-11.

13. Arbelaez AM, Semenkovich $K$, Hershey T. Glycemic extremes in youth with T1DM: the structural and functional integrity of the developing brain. Pediatr Diabetes. 2013; 14: 541-53.

14. Adolfsson P, Riddell MC, Taplin CE, et al. ISPAD Clinical Practice Consensus Guidelines 2018: Exercise in children and adolescents with diabetes. Pediatr Diabetes. 2018;19(Suppl. 27):205- 226.

15. Johnson SR, Cooper MN, Davis EA, Jones TW. Hypoglycaemia, fear of hypoglycaemia and quality of life in children with type 1 diabetes and their parents. Diabetic Med: J Br Diabetic Assoc. 2013; 30:1126-31.

16. Mcgill DE, Levitsky LL. Management of Hypoglycemia in Children and Adolescents with Type

17. 1 Diabetes Mellitus. Curr Diab Rep [Internet]. 2016; 2-7. Available from: http://dx.doi.org/10.1007/s11892-0160771-1

18. Smith $\mathrm{CT}$, Chen $\mathrm{AMH}$, Plake $\mathrm{KS}$, Nash CL. Evaluation of the impact of a diabetes education curriculum for school personnel on disease knowledge and confidence in caring for students. J Sch Health. 2012; 82: 449-456.

19. Brasil. Lei $n^{\circ} 12.982$, de 28 de maio de 2014. Determina o provimento de alimentação escolar adequada aos alunos portadores de estado ou de condição de saúde específica.. Diário Oficial da União. 29 mai 2014; Seção 1. 